\title{
Quantitative Determination of Azelaic Acid in "Propolis" Gel with the Propolis Phenolic Hydrophobic Drug for Treating Acne
}

\author{
Svetlana G. Bobro ${ }^{1}$, Olexandr I. Tikhonov ${ }^{1}$ and Mykola Y. Blazheyevskiy ${ }^{2}$ \\ 1. Department of Cosmetology and Aromatology, National University of Pharmacy, Kharkov 61168, Ukraine \\ 2. Department of Physical and Colloid Chemistry, National University of Pharmacy, Kharkov 61168, Ukraine
}

\begin{abstract}
The aim of the work is to develop and validate the method of quantitative determination of azelaic for treating acne. To determine the AA content in the drug the reversed phase HPLC has been proposed. Specificity of the method was confirmed by chromatograms of placebo and AA. To determine such validation value as precision of the method the AA working standard solution was chromatographed; according to the data obtained the mean value of the content of AA found in percent was calculated. To check accuracy of the results of the method the standard addition method was used. Reproducibility of the method was also checked. Robustness of the method was investigated within changes in the composition of the mobile phase, $\mathrm{pH}$ value of the mobile phase, the column temperature and the mobile phase rate according to the requirements of the State Pharmacopoeia of Ukraine. The method of quantitative determination of AA in a gel according to such indicators as specificity, linearity, precision, accuracy, reproducibility, stability and robustness can be considered valid.
\end{abstract}

Key words: Azelaic acid, acne, propolis, quantitative determination, chromatography.

\section{Introduction}

Acne occupies a special place in dermatological and cosmetic practice. The urgency of the problem of acne for modern scientific and practical medicine is due to the significant prevalence of this disease among people of young and middle age, chronicity, resistance to conventional therapy, disturbance of the patients' psychoemotional state and social adaptation. The majority of domestic and foreign authors think acne to be a serious problem and consider it as a dysfunction of the whole organism.

To treat acne antibacterial agents are often used, i.e. substances that inhibit the growth of bacteria on the skin and sebaceous gland. If the attention is paid to the composition of drugs from acne, most of them contain azelaic acid (AA). This substance exhibits the comedolytic action, i.e. makes sebum more liquid,

Corresponding author: Svetlana G. Bobro, teaching assistant, research field: drug technology. E-mail: sveta_bobro@mail.ru. exfoliates the upper layer of the epidermis, has the anti-inflammatory, antioxidant action, normalizes the lipid barrier of the skin. AA performs important functions in the metabolism of the human body, it is indispensable in the treatment of such diseases of the skin as acne and rosacea.

AA, a saturated dibasic acid (1,7-heptane dicarboxylic acid, $\left.\mathrm{HOOC}\left(\mathrm{CH}_{2}\right)_{7} \mathrm{COOH}\right)$, is a popular active pharmaceutical ingredient used in drugs for treating acne, ATC D10AX03. It is formed during the lipid metabolism and contained in the human body, non-toxic, non-teratogenic, non-mutagenic. AA in vivo and in vitro inhibits proliferation of keratinocytes and normalizes disorder of processes of terminal differentiation of the epidermis when acne appears, accelerates comedolysis of comedones (blackheads). AA reveals the anti-inflammatory effect by inhibition of cell oxidoreductase and suppression of metabolism in non-core granulocytes, as well as generation of free radicals, which are important factors in maintaining 
the activity of the inflammatory process. A part of AA is excreted by the kidneys in an unchanged form; another part is in the form of dicarboxylic $\mathrm{C}_{5}$ and $\mathrm{C}_{7}$ acids formed with AA because of $\beta$-oxidation.

AA is not an officinal medicinal substance, and relatively few methods for its determination are described in the literature. Thus, the content of the active ingredient in the substance can be determined potentiometrically by the nonaqueous titration method, for example, with tetrabutyl-ammonium hydroxide in the medium of pyridine or 2-propanol, or their mixture with benzene [1], or in tert-butanol, acetone or $\mathrm{N}, \mathrm{N}$-dimethylformamide [2]. A simple valid method for quantitative determination of AA in drugs by the nonaqueous titration with sodium methoxide in the presence of thymol blue was suggested [3].

In the scientific literature highly sensitive extraction-photometric methods for determination of AA in various objects, including drugs, for example with methylene blue or methylene violet, are described [4].

For quantitative determination of AA in biological fluids or multicomponent drugs and cosmetic formulations it is recommended to use a selective method of liquid chromatography often in combination with pre-column derivatization [5- 7], gas chromatography with mass spectrometric detection [8-10], or in combination with solid phase extraction of the derivatives obtained and flame ionization detection $[11,12]$. Derivatization methods are more sensitive and allow to quantify small concentrations of AA.

Since treatment medications usually contain AA in relatively high concentrations (usually 15\%-20\%), the high sensitivity of the HPLC method has no practical significance. However, it allows to detect easily the content of AA in multicomponent drugs with sufficient accuracy without additional use of pre-column derivatization as it has been shown in some works [13].

The aim of the work was to develop and validate the rapid method of quantitative determination of AA in a gel in combination with the propolis phenolic hydrophobic drug (PPHD) [14] by the method of reversed phase HPLC.

\section{Materials and Methods}

All solutions were prepared by the volume-weight method using ADV-200 analytical balance and glassware of class 2 .

Test solution: Place approximately $1.0 \mathrm{~g}$ (accurate weight) of the drug in a $50 \mathrm{ml}$ volumetric flask, add $20 \mathrm{ml}$ of the mobile phase, mix thoroughly until a homogeneous mixture, dilute to the volume with the mobile phase, mix thoroughly and centrifuge at the speed of $7000 \mathrm{rpm}$ for $10 \mathrm{~min}$. When required, filter additionally the supernatant liquid obtained through a Teflon membrane filter with pore size of $0.45 \mu \mathrm{m}$ discarding the first $5 \mathrm{ml}$ of the filtrate.

Reference solution: Place approximately $0.08 \mathrm{~g}$ (accurate weight) of the working standard of AA in a $50 \mathrm{ml}$ volumetric flask, dissolve in $20 \mathrm{ml}$ of the mobile phase (ultrasound can be used), dilute to the volume with the mobile phase and mix. Filter the solution obtained through a Teflon membrane filter with pore size of $0.45 \mu \mathrm{m}$ discarding the first $5 \mathrm{ml}$ of the filtrate.

Mobile phase: Place approximately $1.0 \mathrm{~g}$ of ammonium acetate in a $1000 \mathrm{ml}$ beaker, dissolve in y $500 \mathrm{ml}$ of water, add $380 \mathrm{ml}$ of methanol and dilute $\mathrm{pH}$ of the solution obtained to $4.5 \pm 0.1$ with glacial acetic acid by potentiometry. Transfer the solution into a $1000 \mathrm{ml}$ volumetric flask, dilute to the volume with water and mix.

Chromatograph $40 \mathrm{mcL}$ of Reference solution and Test solution each on a liquid chromatograph with a spectrophotometric detector obtaining at least 3 chromatograms under the following conditions:

- SpherisorbODS2 column: $250 \mathrm{~mm} \times 4.6 \mathrm{~mm}$ filled with the sorbent with the particle size of $5 \mu \mathrm{m}$ or the similar one;

- Pre-Column: Symmetry C18 $60 \mathrm{~mm} \times 4.6 \mathrm{~mm}$ 
filled with the sorbent with the particle size of $5 \mu \mathrm{m}$ or the similar one;

- mobile phase degassed by a convenient way;

- the thermostat temperature: $40.0^{\circ} \mathrm{C}$;

- the rate of the mobile phase: $1.0 \mathrm{ml} / \mathrm{min}$;

- detection: at the wavelength of $230 \mathrm{~nm}$.

The chromatographic system is considered suitable if the following conditions are performed:

- the efficiency of the chromatographic system calculated according to the AA peak must be not less than 1000 theoretical plates;

- the peak symmetry factor of AA must be not more than 2.0;

- the relative standard deviation of peak areas of AA must meet the requirements of 2.2.46 (SPhU 1.2).

The content of AA $(\mathrm{g})$ in $1.0 \mathrm{~g}$ of the drug is calculated by the formula:

$$
Y=\frac{S \cdot m_{0} \cdot 50 \cdot P}{S_{0} \cdot 50 \cdot m \cdot 100}=\frac{S \cdot m_{0} \cdot P}{S_{0} \cdot m \cdot 100},
$$

Where, $S-$ is the average peak areas of AA calculated from chromatograms of the Test solution;

$S_{0}$ - is the the average peak areas of AA calculated from chromatograms of the Reference solution;

$m_{0}-$ is the sample weight of the AA working standard, $\mathrm{g}$;

$P-$ is the content of the active ingredient in the AA working standard, \%;

$m-$ is the sample weight of the drug, g.

The content of AA in $1 \mathrm{~g}$ of the gel should be from 0.076 to $0.084 \mathrm{~g}$.

\section{Results and Discussion}

Determination of the content of AA in the drug is proposed to carry out by the method of reversed phase HPLC under the following conditions:

- SpherisorbODS2 column: $250 \mathrm{~mm}$ x $4.6 \mathrm{~mm}$ filled with the sorbent with the particle size of $5 \mu \mathrm{m}$ or the similar one;

- Pre-Column: SymmetryC18 $60 \mathrm{~mm}$ x $4.6 \mathrm{~mm}$ filled with the sorbent with the particle size of $5 \mu \mathrm{m}$ or the similar one;

- mobile phase degassed by a convenient way;

- the thermostat temperature: $40.0{ }^{\mathrm{O}} \mathrm{C}$;

- the rate of the mobile phase: $1.0 \mathrm{ml} / \mathrm{min}$;

- detection: at the wavelength of $230 \mathrm{~nm}$.

Under these conditions the peak of AA is rather separate from the excipients of the gel.

Specificity of the method was confirmed by chromatograms of placebo and AA (chromatograms are given in Figs. 1-3). It is shown that in the chromatogram of the placebo solution there are no peaks with retention time, which coincides with the retention time of $\mathrm{AA}$.

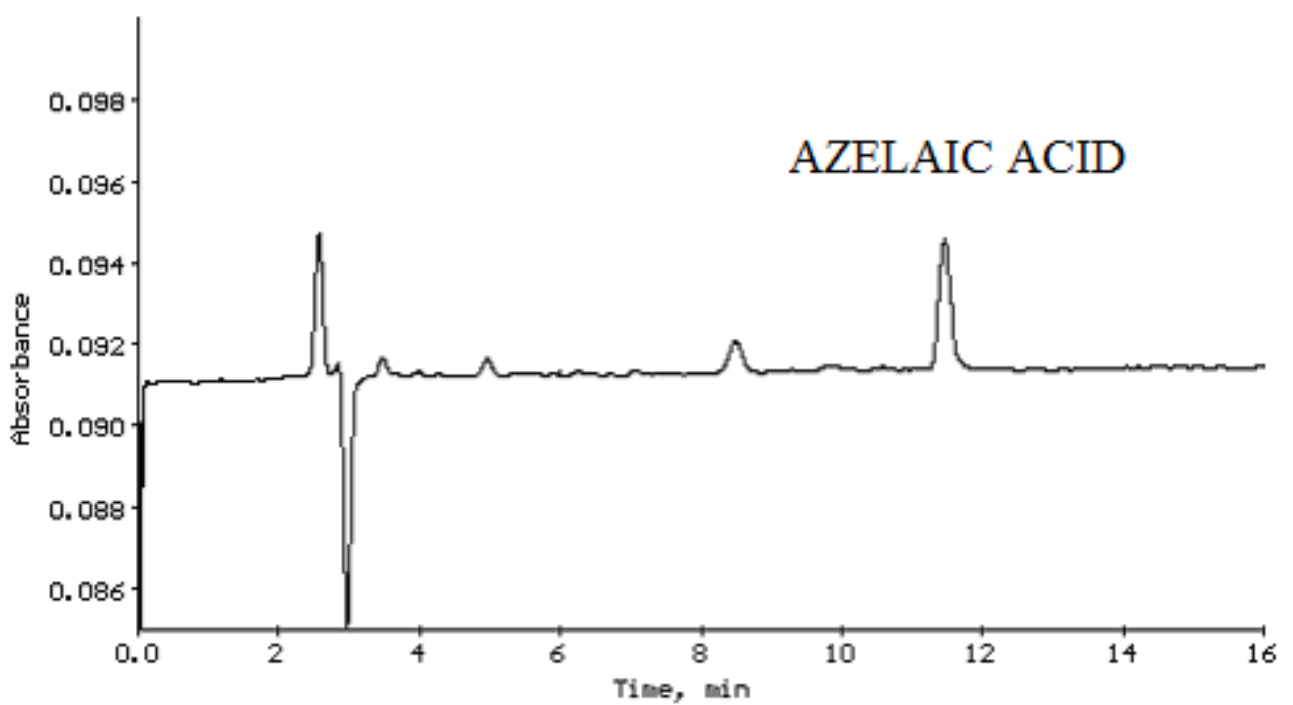

Fig. 1 The chromatogram of the working standard solution of AA. 


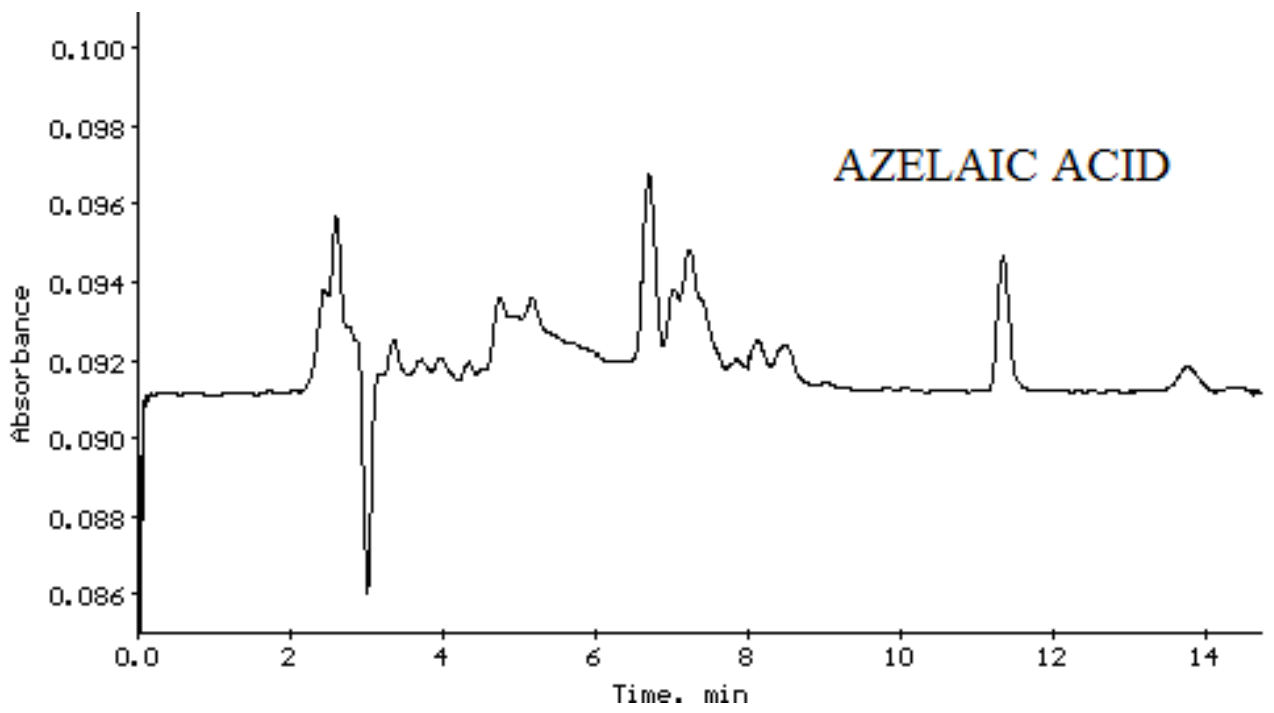

Fig. 2 The chromatogram of the test solution (drug).

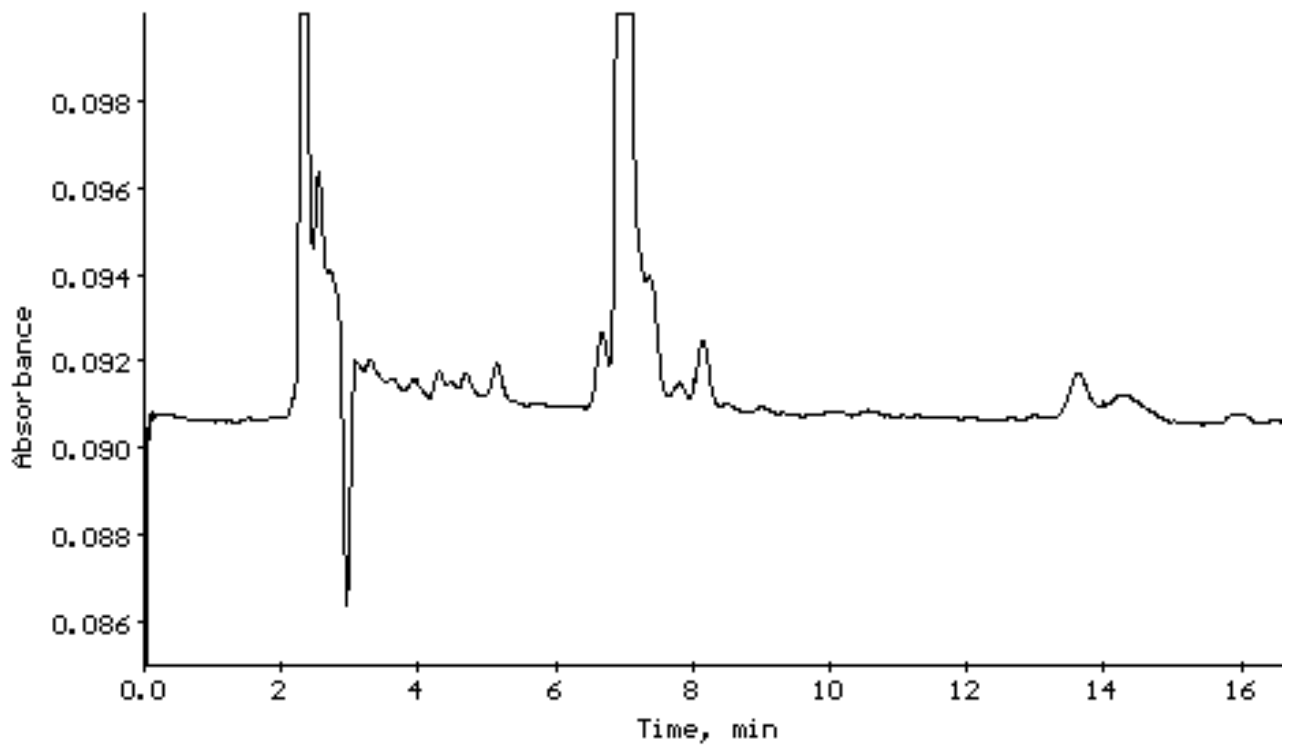

Fig. 3 The chromatogram of the placebo solution.

Chromatographic analysis was performed on a Hewlett Packard 1050 Agilent Technolog microcolumn liquid chromatograph (Germany). Automatic sampling was provided by the programmed autosampler. The $\mathrm{pH}$ value of the solutions was measured by the direct potentiometry method on an I-130 laboratory ion meter (RSC “Analytpribor”) with the help of a glass electrode of ESL 43-07 type; a silver chloride electrode saturated with potassium chloride was used as a reference electrode.

Processing of the experimental data was performed using Excel spreadsheet of the Microsoft Office
Professional 2003 software package.

Under the conditions proposed, linearity of the dependence of the detector response on the concentration of AA was checked. To obtain the data about the linear dependence solutions of AA containing $80 \%, 90 \%, 100 \%, 110 \%$ and $120 \%$ of the label claim were prepared. The solutions were chromatographed three times. According to the data obtained the graph of the dependence of the peak area (Y) on the content of AA in $\mathrm{mg} / \mathrm{ml}$ was plotted (Fig. 4). Characteristics of the linear dependence $(\mathrm{Y}=\mathrm{a}+$ bX) obtained are given in Table 1. 


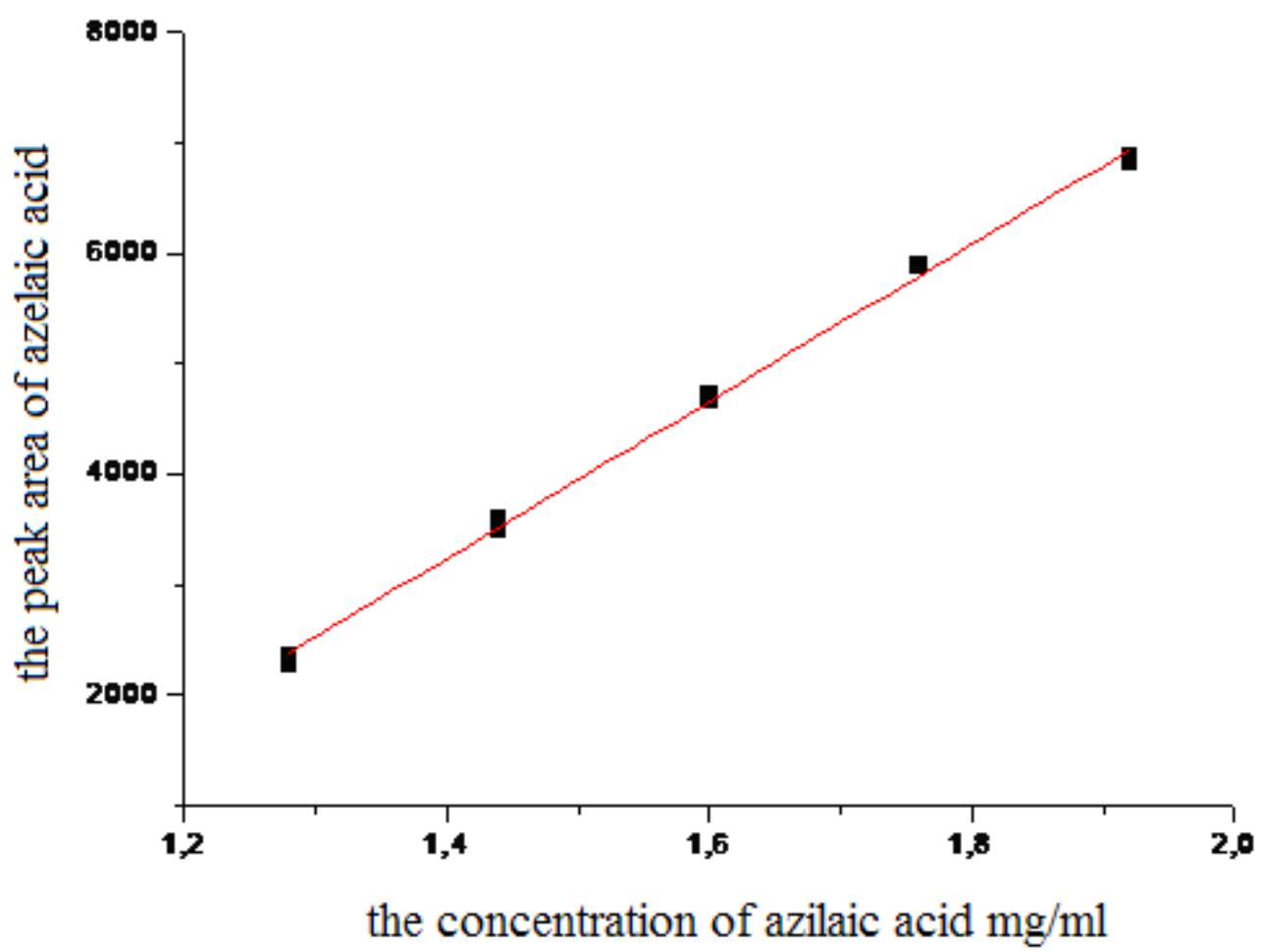

Fig. 4 The dependence of the detector response (peak area) on the concentration of AA.

Table 1 Characteristics of the linear dependence $\mathrm{Y}=\boldsymbol{a}+$ bX.

\begin{tabular}{ll}
\hline$A$ & $-7 \cdot 10^{3}$ \\
\hline $\mathrm{S}_{a}$ & $1 \cdot 10^{3}$ \\
$B$ & $71.1 \cdot 10^{2}$ \\
$\mathrm{~S}_{\mathrm{b}}$ & $0.7 \cdot 10^{2}$ \\
$R$ & 0.9989 \\
Detection limit (DL) & $0.04 \mathrm{mg} / \mathrm{ml}$ \\
Quantification limit (QL) & $0.12 \mathrm{mg} / \mathrm{ml}$ \\
\hline
\end{tabular}

To determine such validation value as precision of the method the AA working standard solution was chromatographed, and then according to the data obtained the mean value of the content of AA found in percent was calculated (Table 2).

To check accuracy of the results of the method the standard addition method was used. The results obtained are presented in Table 3.

Reproducibility of the method was also checked. The quantitative determination of the AA content in the gel studied was performed in different days. The results obtained are presented in Table 4.

The data obtained show that the method is stable and reproduced in time.
Robustness of the method was investigated within changes in the composition of the mobile phase, $\mathrm{pH}$ value of the mobile phase, the column temperature and the mobile phase rate according to the requirements of the State Pharmacopoeia of Ukraine (SPhU) 1.2, Art. 2.2.46. The results obtained for efficiency of the chromatographic system, asymmetry of the chromatographic peak and RSD values calculated for AA show that minor changes in the above parameters have no significant effect on chromatographic characteristics of the method. The method can be considered robust.

Standards for the System Suitability Test were introduced to the method developed to confirm its efficiency and achieve the correct results when reproducing the method.

Therefore, we have developed a simple and rather selective method for quantitative determination of AA in the gel, which includes PPHD, using reversed phase HPLC. According to such validation indicators as specificity, linearity, precision, accuracy, reproducibility, stability and robustness it can be considered valid; 
Table 2 The results of analysis of azelaic acid in model solutions.

\begin{tabular}{llc}
\hline Concentration of azelaic acid in the working standard solution, $\mathrm{mg} / \mathrm{ml}$ & Found of azelaic acid, $\mathrm{mcg} / \mathrm{ml}$ & Found, $\%$ \\
\hline 1.200 & 1.205 & 100.42 \\
1.350 & 1.353 & 100.23 \\
1.500 & 1.499 & 99.94 \\
1.650 & 1.647 & 99.83 \\
1.800 & 1.796 & 99.77 \\
Mean value & & 100.04 \\
RDS, \% & & 0.34 \\
\hline
\end{tabular}

Table 3 The results of analysis of azelaic acid in model solutions without and with addition of AA.

\begin{tabular}{|c|c|c|c|}
\hline \multirow{2}{*}{ Addition of azelaic acid, mg } & \multicolumn{2}{|c|}{ Found of azelaic acid, mg } & \multirow{2}{*}{-Found, \% } \\
\hline & With addition & Without addition & \\
\hline- & - & 1.65 & - \\
\hline 0.40 & 2.00 & 1.60 & 97.2 \\
\hline 0.80 & 2.53 & 1.73 & 104.6 \\
\hline 1.20 & 2.90 & 1.70 & 102.8 \\
\hline 1.60 & 3.24 & 1.64 & 99.1 \\
\hline Mean value & & & 100.9 \\
\hline
\end{tabular}

Table 4 The results of quantitative determination of $\mathrm{AA}$ in model solutions in different days.

\begin{tabular}{lllll}
\hline \multirow{2}{*}{$\%$ of the nominal content } & Convergence, mean, \% \pm RSD, \% & \multicolumn{3}{c}{ Precision (reproducibility) } \\
\cline { 3 - 5 } & & Day 1 $\% \pm$ RSD, \% & Day 3 \\
\hline 80 & $100.2 \pm 0.1$ & $100.05 \pm 0.09$ & $100.3 \pm 0.2$ & $100.11 \pm 0.06$ \\
100 & $100.03 \pm 0.08$ & $99.98 \pm 0.05$ & $100.2 \pm 0.3$ & $99.9 \pm 0.1$ \\
120 & $100.2 \pm 0.2$ & $100.0 \pm 0.3$ & $99.95 \pm 0.05$ & $100.1 \pm 0.1$ \\
\hline
\end{tabular}

therefore, it can be used in pharmaceutical analysis of the gel for determining the AA content.

\section{Conclusions}

The conditions have been offered, and the method for quantitative determination of AA in the gel with PPHD has been developed using reversed phase HPLC with a spectrophotometric detector.

The validation procedure of the method has been carried out according to such indicators as specificity, linearity, precision, accuracy, reproducibility, stability and robustness. Detection and Quantification limits of AA in "Propolis" gel have been determined.

\section{References}

[1] Turgut, G., Esma, K., and Güleren, Ö. 1988. "Titrationsinnon-aqueousmedia. Part XII. Potentiometric titrations of symmetrical aliphatic dicarboxylic acids and some of their binary mixtures in different media."Analyst
113: 1017-21.

[2] Aslan A., Aslan, A., Erdogan, Y., Demirbas, A., and Karslioglu, S. 1997. "Potentiometric Titration of Some Dicarboxylic Acids in Non-equeous Media." Pharmazie 52 (4) 309-10.

[3] Khazaeli, P., and Keramati, M. 2004. "Non-aqueous Titration for the Determination of Azelaic Acid: Introduction and method validation." J. Kerman Univer. Med. Sci. 11 (3) 5-8.

[4] Medikondu, K., Jayaprakash, M., and Vijayabhaskara, R. T. 2010. "Spectrophotometric Determination of Azelaic Acid In Pharmaceutical Formulations/Medikondu Kishore.” J. Pharm. Res. 3 (12) 3090-2.

[5] Ferioli, V., Rustichelli, C., Vezzalini, F., and Gamberini, G. 1994. "Determination of Azelaic Acid in Pharmaceuticals and Cosmetics by RP-HPL Cafterpre-column Derivatization. "Il Farmaco. 49 (6) 421-5.

[6] Scalia, S. Assay, S., Scalia, A., Bianchi, S., and Guarneri, V. M. 1997. "Of underivatized azelaic acid in pharmaceutical and cosmetic products by HPLC." Pharmazie. 52 (12) 929-31.

[7] Gatti, R., Andrisano, V., Di Pietra, A. M., and Cavrini, V. 
1995. "Analysis of aliphatic dicarboxylic acids in pharmaceuticals and cosmetics by liquid chromatography (HPLC) with fluorescence detection." J. Pharm. Biomed. Analysis 13 (4-5): 589-95.

[8] Garelnabi, M., Litvinov, D., and Parthasarathy, S. 2010. "Evaluation of Agas Chromatography Method for Azelaic Acid Determination in Selected Biological Samples." N. Amer. J. Med. Sci. 2 (9) 397-402.

[9] Ma, Y., Chun, J., Wang, S., and Chen, F. 2010. "Biotechnol Allelopathic Potential of Jatrophacurcas." Afric. J. 10 (10): 11932-42.

[10] Št'ávová, J., Beránek, J., Nelson, E. P., Diep, B. A., andKubátová, A. 2010. "Limits of Detections for the Determination of Mono-and Dicarboxylic Acids Using Gasandliquid Chromatographic Methods Coupled with Mass-Spectrometry.” Anal. Technolog. Biomed. Life Sci.
879 (17-18): 1429-38.

[11] Panavaite, D., Adomavičiūtè, E., and Vičkačkaitè, V. A. 2006. "Anovel SPME fibre for fatty acid determination." Chemija. 17 (4) 61-6.

[12] Urbánek, M., Pospíšilová, M., Polášek, M., and Šícha, J. 2002. "Determination of azelaic and sorbic acidin pharmaceduticals by capillary electrophoresis." Chromatogr. 55 (5-6) 333-7.

[13] Mansour, A. M., and Ibrahiem, M. M. 2002. "Simultaneous Determination of Azelaic and Benzoic Acids Intopical Preparations by Liquid Chromatography." Chromatographia. 55 (7-8): 435-7.

[14] Propolis phenolic hydrophobic drug (PPHD) - PC No. UA/4505/01, Order of the Ministry of Public Health of Ukraine No. 337 from 07.06.2011 (in Ukrainian). 